\title{
Case Study: Optimization of End-of-line Packaging in Fishery Industry
}

\author{
Tanja Kerezovic, Gabor Sziebig, Member, IEEE
}

\begin{abstract}
Application of automation in SMEs working in fish industry is not in advanced state as in other fields of production and manufacturing industries. Due to expensive equipment, high labor costs of engineers required to implement such features into production lines, and low-quantity contracts, introduction of automation technologies is in large part of these companies is absent.

This paper analyses introduction of new technologies into an example company's end-of-line packaging and palletizing line, trying to find and ultimately, suggests a fully automated packaging and palletizing solution. This company is based in Northern Norway, whose main production line handles salmon processing. Due to recent expansion of the company, new facilities are being built, and the company plans to use modern technologies to aid the quality and speed of processing of the fish, thus reducing manual labor and associated costs.
\end{abstract}

\section{INTRODUCTION}

Food industry is a wide and diverse sector, both in terms of the products and the structures. Some of the sectors included in this industry are:

- Agriculture and aquaculture -concerned with growing crops, raising livestock and seafood

- Manufacturing of machinery, seeds, agricultural chemicals

- Food processing-processing of fresh products into packed, canned products, frozen or pre-made food

- Food service and retailing-including catering, restaurants, shops, markets

- Distribution, logistics and transportation of food and food products

- Animal feed

- Beverage industry

With its fjords and islands, Norwegian coastline stretches more than $83000 \mathrm{~km}$ in length. This provides perfect environment and conditions for fisheries and aquaculture. Although Norway is 12th largest country in the world by production of fish [1], considering both catches and aquaculture, it is second largest fish processor in the world [2]. When it comes to EU, Norway is the number one supplier or fish and aquaculture products and account for

T. Kerezovic is with the Department of Industrial Engineering, UiT The Arctic University of Norway, Postboks 6050 Langnes, 9037 Tromsø, Norway (e-mail: tanja.kerezovic@gmail.com)

G. Sziebig with the Department of Industrial Engineering, UiT - The Arctic University of Norway, Postboks 6050 Langnes, 9037 Tromsø, Norway (e-mail: gabor.sziebig@uit.no). more than one-fourth of the total fish product imports, both by volume and by value. Throughout the Norwegian history, fisheries and aquaculture played key social and economic role. It has been the basis for settlement and employment along the Norwegian coast and a major contributor to rural development, especially in the remote areas. The processing industry in Norway, like in the EU, mostly consists of a large number of small and medium-sized enterprises that are scattered along Norwegian coastline.

Food industry, and SMEs in general, rely heavily on manual labor. Labor costs can account up to 50 percent of the product cost. What's more, labor productivity in fish industry is among lowest in the EU. This means that these industries are not using its employees effectively, and in high-wage countries like Norway, it creates major concern and is making Norway less competitive.

High operating costs are forcing some of the producers to relocate their production to lower- cost countries in the EU [3]. Additionally, negative exchange trends and increasing difficulties to access the EU market are impacting this industry as well. To respond to these changes, fish industry and food industry as general, recognized the opportunity provided by modern technology. Since food industry is highly labor-dependent, by automation at least some of these activities, profitability, as well as productivity, would be highly influenced. But consisting mainly of SMEs, these industries have very limited resources. Financial resources are just one of the resources SMEs lack when implementation of automation is concerned. Another big limitation is lack of engineering resources. Engineers in the food industry are usually focused on daily operations, and lack time to develop new ideas, skills and do the needed research.

As previously stated: on one hand - companies in the fish industry need to increase the automation level in their production, but on the other hand - they have very limited financial and engineering resources to achieve this. That is one of the issues that this paper is focusing on, trying to prove that it is possible to obtain both, to a certain degree.

This paper takes a study case of a company in Northern Norway, Brødrene Karlsen AS, involved in fish processing sector of food industry. By focusing on their salmon processing line, the customized automated solution for the end-of-line packaging is developed in order to show how automation can help SMEs to decrease dependence on manual labor, while still trying to maintain low investment costs. In addition, the paper investigates the market offers in the sector of end-of-line palletizing and proposes suggestions for further automation on a higher level. 


\section{RELATED RESEARCH}

\section{A. Current State of Automation in Food Processing Industry}

In the field of food processing industry, the automation used is composed of task specific machines and robotic systems. Task specific machines are usually fixed automation machinery, which is used for a dedicated production line, while robotic systems are used in areas where more flexibility is needed.

Food industry was slow to adopt new technologies, including robots. Their use in this industry started a little bit longer than a decade ago, and still represents around $2 \%$ of the robot population in all industries [4].

Figure 1. Estimated annual supply of industrial robots by main industries 2011-2014 [5]

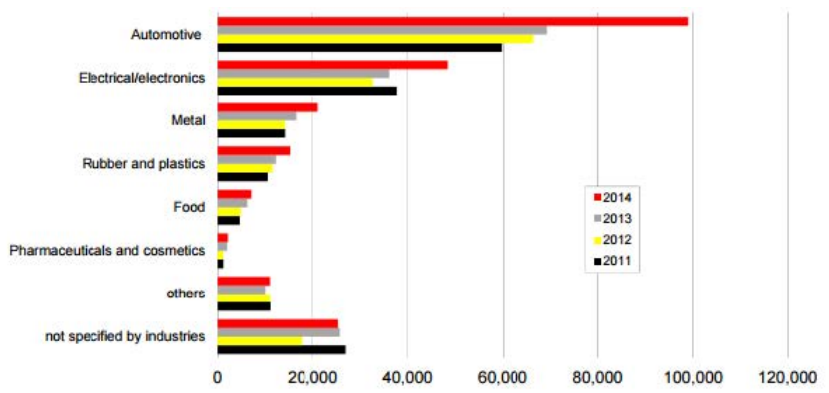

As can be seen from the Figure 1, food and pharmaceutical industries have the lowest number of robots installed. Although growing trend is observed in these industries in last years, it is not nearly as optimistic as with other industries, such as automotive or electronic industry.

Robots are the future of any industry, and food industry is starting to open up to these possibilities. If the forecasts are correct, the annual supply of robots in the years to come will just keep growing (Figure 2).

Figure 2. Worldwide annual supply of industrial robots 2000-2014 with forecast for 2018 [5]

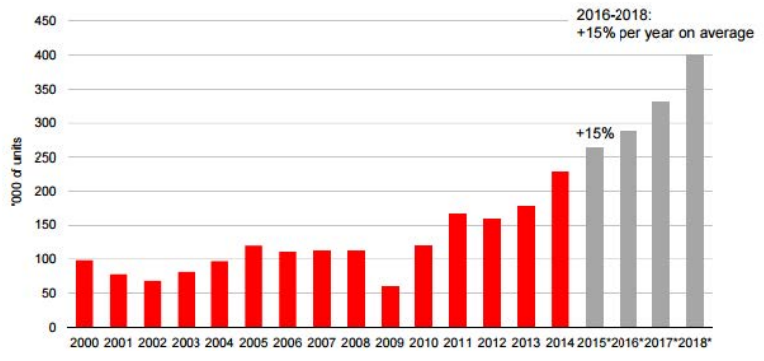

\section{B. Automation Challenges in Food Industry}

Previously it was mentioned that the food industry has been slow to adopt new automation technologies. This is mainly due to some constraint that are not present in other industrial sectors.

In previously mentioned survey the automation level warried widely across plants of different age groups and different annual productions. The analysis of the survey results has shown generally known fact: plants with smaller production level per year (in this case less than 100000 tons) are less automated than those with higher volume production.
The obstacles for implementation of new technologies appear to be [6]:

- For smaller volume production plants those are time and cost;

- For larger plants, it is cost and management commitment.

This comes as no surprise, since most of the companies in the food industry fall into the category of SMEs. It was said in the Chapter 1 that these small businesses have very limited resources: financial and engineering. Therefore, the cost obstacle represents the lack of financial resources and time represents the lack of engineering resources to investigate and implement new solutions. That indicates one of the reasons why food industry, and fish industry in particular, still heavily relies on manual labor. Other reasons include [7] \& [8]:

- The flexibility of the human worker: human beings are able to handle complex object manipulation with ease, through combination of sensor and hand capabilities (hand-eye coordination) and accumulated experience. They are excellent at evaluating situations, while robots have limited decision making ability.

- Another reason is the mobility and agility of humans: dedicated machinery can be built to suit the task, and robots have limited motion and relatively small working envelope. Therefore, the traditional automated systems are incapable of high flexibility needed to cope with variation in products and production demands.

- Properties of the food product are not the same as other engineering materials. Most of the food products cannot be adequately geometrically described, where some of the reasons include:

oThey vary in shape, texture, size,

oThey are susceptible to forces applied,

oThey are affected by time spent in the processing stage, as well as environmental conditions: temperature, humidity and pressure,

oThese products are susceptible to hazards,

oProducts are often delicate and non-rigid.

- The product deforms considerably based on the type and way of handling. At the moment there is not enough end-effectors for manipulating these types of products as well as handling strategies.

The listed reasons for use of manual labor can be translated to the requirements modern automation needs to meet. Apart from these requirements, when it comes to application of robotics and other types of automation in the food processing facilities, there are other issues to consider [7] \& [9]:

- These automation systems have been developed for the manufacturing industry and as such cannot crossover 
into food industry without considerable changes. One of those changes are related to utmost importance of hygiene and cleanliness of machinery in the food industry.

- Machines or robotic systems that are employed in the food industry need to fulfil construction requirements regarding materials they are made of, which are limited to stainless steel and few plastics, and the construction that needs to be waterproof. They need to be able to withstand high pressure wash-down, often with chemical cleaners, with all external parts accessible for inspection and manual cleaning.

- Automated system is needed to be able to work in environments with low and high temperatures and humidity.

- In many cases these companies need automation solution that can be moved around and can work with existing manual operations, which contradicts the most of the current automation systems, where they are physically separated from people and limited ability of coping with frequent changes in the manufacturing process.

- Robot manufacturers and system integrators often have not so adequate understanding of the specific challenges food industry faces and requirements they, therefore, have. These requirements and operating conditions vary heavily compared to other sectors that need automation or robotic solutions. This often results in wrong product offers, or not enough of appropriate models.

\section{PRoBlem ANALYSIS}

End-of-line palletizing is a term that includes all actions and processes included in secondary and tertiary packaging. For different companies, end-of-line represents different processes. At Brødrene Karlsen, end-of-line activities include:

\section{Loading the fillets into pre-opened boxes}

2. Weighting, closing, taping and labelling the boxes

3. Guiding boxes through metal detector

4. Palletizing the products.

The Company has different types of main products, and the layout of the production line slightly changes depending on the product being manufactured. The products that demand a change in the processing line are:

- Single fillets, frozen in single freezer

- Fresh fillets, packaged in chains and frozen in the tunnel freezer

- Fresh tail part of the fillet, single packed and frozen in the tunnel freezer

Freezer is located in a processing facility, and therefore can be a part of the processing line. Single fillets go into this type of freezer where they are moved on the conveyor and frozen within one hour, so called "fast freezing". By using this technique, molecules inside food do not have time to form large ice crystals, and therefore the fish meat keeps its moisture. Needless to say that this type of freezing provides better quality of the products.

Other type of freezer is the tunnel freezer. Inside the tunnel freezer, cold air is pushed at high velocity in order to freeze the products as fast as possible. This freezer is used for all products, except previously mentioned single fillets. Products like: fillets in chains, tails and all by- products are frozen in this freezer. Products are packed into boxes, and boxes are placed onto pallets, but between every row of boxes, a slip sheet is placed. These plastic sheets are placed between the rows in order to increase the air flow between boxes and consequently, freeze products faster. In order for products to be frozen properly, they have to remain in the tunnel freezer between 24 and 48 hours, which depends on the product. Depending on the product being manufactured, the layout of the production line slightly changes to accommodate the difference in production steps. This change happens 2-3 times a week, depending on the orders. Production of by-products is independent of type of main product made, since the by-products are the same in all types of fillets produced. It is important to note that tails are not always being produced. Mainly it depends on the customer requirements.

- During the production of single fillets, tails are always separated. This is because single fillets frozen in the single freezer are high quality products and tails are not. Tails are packed and sold to different customer.

- During production of chain fillets, sometimes the tails are separated and sometimes they are one of the fillets in chains. Again this depends on the customers, some of them want higher quality fillets and don't want the tail parts, while others want lower price of the fillets and therefore include tail parts.

In order to analyze the flow of different products in the packaging are, flow chart has been made (Figure 3). The chart only includes the steps in the packaging and palletizing area, i.e. the steps after the vacuuming and freezing station.

The red dot represents the flow of single fillets. They are vacuum packed and frozen in the single freezer and are coming to the sorting station frozen and ready to be packed. The sorting station is used to separate fillets for the in-feed to the grader, but also as a quality check in case of improper vacuum or fillets not being frozen enough.

The green dot represent the flow of fresh fillets packaged in chains, as well as the flow of packaged tails, since these two products are packaged fresh and have the same flow through the packaging line.

Orange dot represents the by-products. They are packaged during production and arrive to palletizing station packed in boxes. These boxes are already labelled, and therefore they need to go through metal detector and be palletized.

The important thing to note is the flow of products during palletizing. Since single fillets are already frozen, they are 
packed into boxes, palletized and moved to cold storage. On the other hand, rest of the products are palletized with slipsheets and are left in the tunnel freezer for 1 to

2 days. After that, boxes with these products need to repalletized, i.e. palletized without slip- sheets.

Another thing to pay attention to, on the flow diagram, are the colors of different stages during end-of-line palletizing. Yellow squares represent states, while other colors represent activities that involve operators or machinery. The states in the yellow squares are: single freezer, tunnel freezer and cold storage.

Figure 3. Product flow in packing and palletizing area

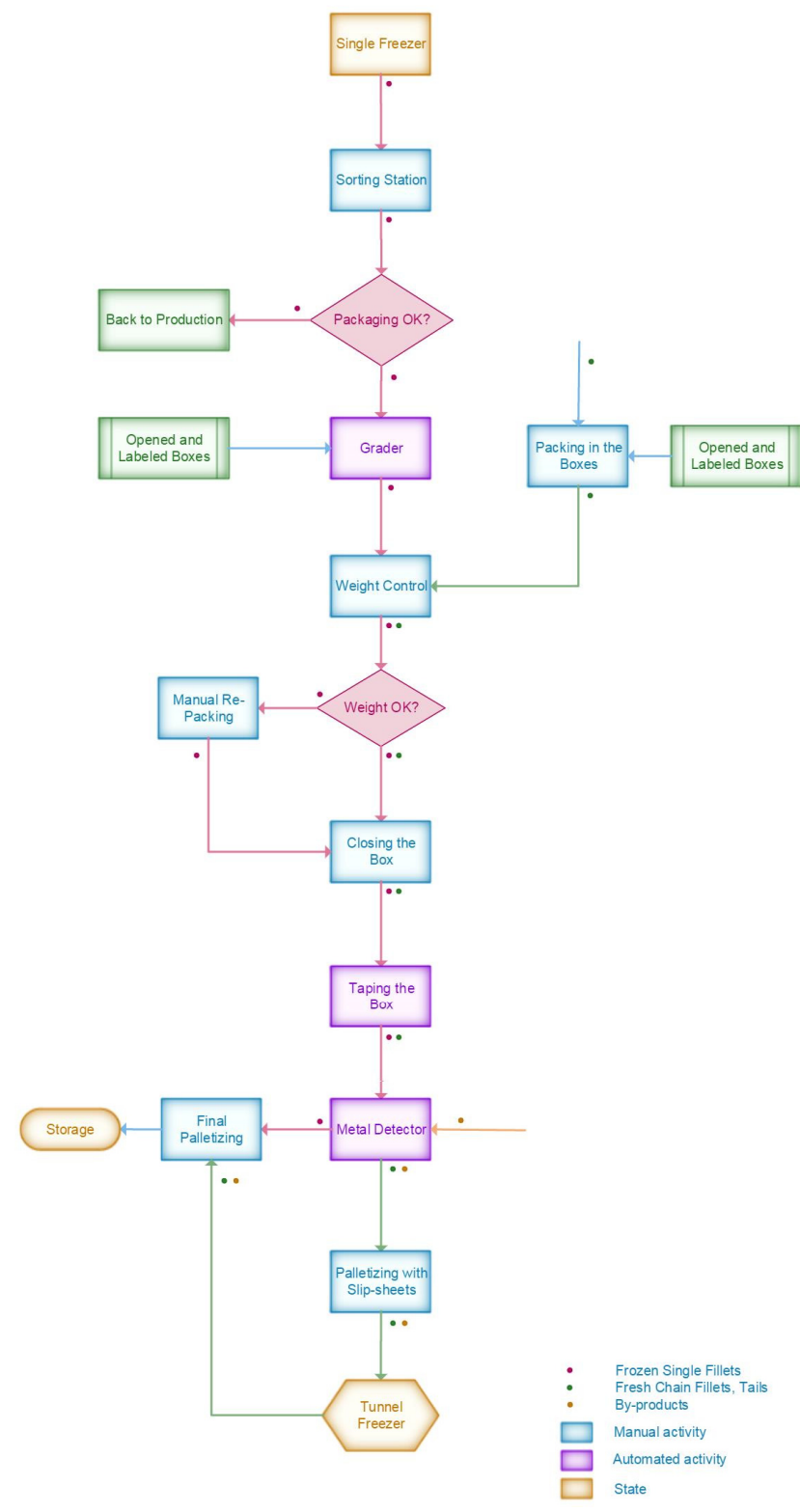

Purple squares represent activities that are already automated:

- Grading the fillets into different boxes is done by automatic grader
- Taping the boxes is done by using automated taping machine

- Metal detector is used to automatically inspect the boxes for foreign metal pieces

Final category are manual activities, which are represented by blue boxes on the flow chart. For frozen fillets, these activities are:

- Sorting frozen fillets on the sorting station performed by one operator

- Opening and labelling the boxes - operated by one worker

- Serving the grader: placing empty box under grading units, removing full boxes and controlling the weight by placing them on the check weighting done by one operator

- Closing the boxes and sending them to taping machine - performed by one operator

- Palletizing - done by one worker.

If the production of fresh fillets is observed, manual activities involve four manual workers:

- Opening and labelling the boxes - one operator

- Packing the fillets in chains into boxes and weighting them - one operator

- Closing the box and sending them to taping machine - one operator

- Palletizing the boxes with slip sheets - one operator.

These activities are done in cold environment and include repetitive work. Palletizing, apart from being repetitive, includes lifting heavy weights since some of the boxes can be up to $20 \mathrm{~kg}$. This increases the risk of repetitive strain injuries (RSI) and especially lower back strains.

The goal of this paper is to automate as much of these manual activities as possible. Ideally, all of the activities could be automated, but there are some constraints that need to be taken into account.

\section{Proposed SOLUTION}

The preliminary design of the line is developed. The line consists of two main flows: (Also shown on Table 1.)

- Flow of the main products: depending on type of the production, these can be single fillets or fillets in chains

- Flow of by-products

There is no need for packing the by-products into boxes, since that is done in processing area. They arrive to the endof-line already packed, and therefore they move forward to palletizing, going through metal detector on their way.

The flow of the main products is further divided into two lines:

- Top line represents the solution for packing of fillets in chains into boxes 
- The bottom line represents the solution for packing single fillets into boxes

The only difference in these two lines is the way of placing the products into boxes, and the activities that follow are the same. For that reason, it is necessary to purchase just one of the following machines:

- Checkweigher

- Case sealer machine

- Metal detector

TABLE I. SOLUTIONS FOR STEPS IN END-OF-LINE

\begin{tabular}{|c|c|c|}
\hline & Single fillets & Fillets in chains \\
\hline $\begin{array}{l}\text { Opening the } \\
\text { boxes }\end{array}$ & \multicolumn{2}{|c|}{ Case opening machine } \\
\hline Case filling & Grader & Gravity case packer \\
\hline Weight control & \multicolumn{2}{|c|}{ In-line checkweigher } \\
\hline $\begin{array}{l}\text { Closing and } \\
\text { taping the boxes }\end{array}$ & \multicolumn{2}{|c|}{ Automatic random case sealing machine } \\
\hline Labelling & $\begin{array}{c}\text { Print \& } \\
\text { Apply } \\
\text { Labeller }\end{array}$ & Standard in-line labeller \\
\hline Palletizing & \multicolumn{2}{|c|}{ Gantry robot or Articulated arm robot } \\
\hline
\end{tabular}

Based on the proposed design the flow of single fillets would be as follows:

- Single fillets arrive on the sorting station and after being inspected, they proceed to the grader.

- Case opening machine opens the boxes and feeds them to the buffering conveyor before the grader.

- Once the grader gives the signal that the box is filled and it needs to be replaced with the empty box, that signal is forwarded to the labelling unit. The labeller prints out the appropriate label and places it on the box.

- The box is discharged for the buffering conveyor and based on the proposed solution for serving the grader, it is automatically placed under the appropriate grading unit.

- Full box after the grader continues to the weighting unit. If the weight is inside the defined limits, it passes through, otherwise it is rejected to the rework station.

- Boxes with correct weight are fed into automatic closing and sealing machine.

- After closing and taping the boxes, they go through metal detector and go towards buffering conveyor of palletizing station.

- At the end of the buffering conveyor there is a barcode scanner, which informs the palletizing robot which box arrived, therefore it knows on which pallet to place it.

- The robot grabs the box and places it on the appropriate pallet.

The flow of fillets in chains is:
- Fillets in chains are fed into the gravity case packer from the top conveyor.

- Case opening machine opens the boxes and afterwards the labeller puts a label.

- Cases are guided towards the gravity packer which fills the boxes with products.

- Filled boxes are brought up by the inclined conveyor and guided towards the checkweigher.

- The following steps are the same as in the previous case.

The propose solution is shown in Figure 4, the interchange between different production lines would be quite easy, and with that the flexibility requirement is fulfilled.

Figure 4. Proposed solution

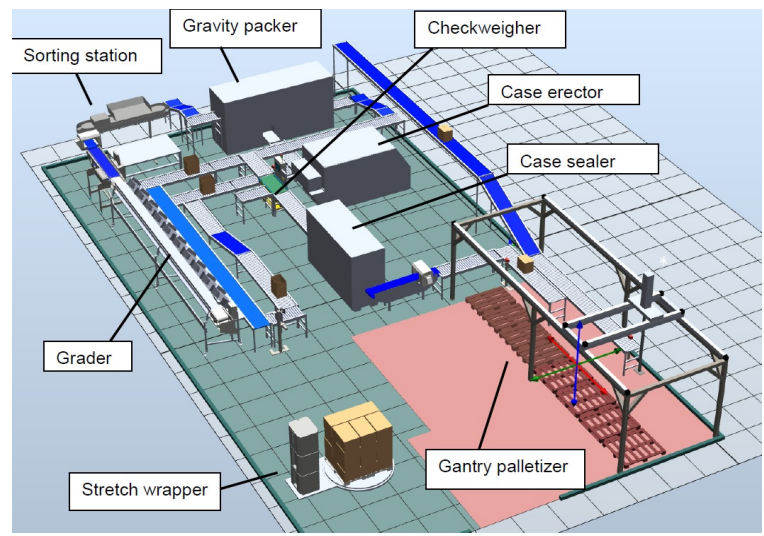

By using this layout the space requirement if fulfilled with satisfactory product flow, as well as worker flow and safety. The fork-lifter is able to safely and easily access the pallets and stretch wrapper.

Since the positions of the pallets was a great concern, simulation helped to visualize and find the optimal solution. By using the articulated robot, much of the space would be lost on fork-lift approach paths. Furthermore, if the Company wishes to invest into automated pallet exchange system in the future, by using this layout that is possible.

The through put of the current line at Brødrene Karlsen is 250 cases/hour and the tails production is additional 10 cases/hour, which lead to $4.3 \mathrm{cases} /$ minute as a speed of the line. The proposed line has a speed capacity as the slowest machine in the line, and in the simulated solution case erecting and case sealing machine speed depends on the box sizes, which is in the range between 18 and 20 cases/minute for the case sealer.

\section{CONCLUSION}

Small and medium companies, such as Brødrene Karlsen, will face many challenges in the future. This can be deducted from the developing trends that influence food and fish industry, as well as higher requirements for food safety and contamination. To remain competitive, they will need to increase the productivity and decrease their dependence on 
manual labor. The way forward is seen in application of automation in the processing and packaging lines. While the labor costs are increasing every year, the prices of automation and electronics are decreasing.

This paper had the main goal to propose an automated and functional solution for end-of-line packaging and palletizing in the company. By observing final solution, it can be seen that these goals were achieved.

\section{ACKNOWLEDGMENT}

The research was supported by the TARGET Project, which is supported by the European Union Northern Peripheries and Arctic (NPA) Programme under Priority 1: Innovation, Research and Technology Development. The NPA Programme has been established to help peripheral and remote communities on the northern margins of Europe to develop their economic, social and environmental potential.

\section{REFERENCES}

[1] European Commission, "Facts and figures on the common Fisheries Policy," 2016.

[2] ESCIP Consortium, "The competitive position of the European food and drink industry," 2016.

[3] Food and Agriculture Organization of the United Nations, "Fishery and Aquaculture Country Profiles: The Kingdom of Norway," 1 January 2011. [Online]. Available: http://www.fao.org/fishery/facp/NOR/en. [Accessed 18 May 2016].

[4] N. P. Mahalik and A. N. Nambiar, "Trends in food packaging and manufacturing systems and technology," Trends in Food Science \& Technology, vol. 21, pp. 117-128, 2010.

[5] Internation Federation of Robotics, "Industrial Robot Statistics," 2015. [Online]. Available: http://www.ifr.org/industrial-robots/statistics/. [Accessed 26 February 2016].

[6] S. V. Ilyukhin, T. A. Haley and R. K. Singh, "A survey of automation practices in the food industry," Food Control, vol. 12, no. 5, pp. 285296, 2001.

[7] D. G. Caldwell, S. Davis, R. J. Moreno Masey and J. O. Gray, "Automation in Food Processing," in Springer Handbook of Automation, 1st ed., S. Y. Nof, Ed., Springer-Verlag Berlin Heidelberg, 2009, pp. 1041-1059.

[8] G. Purnell, "Robotic Equipment in the Meat Industry," Meat Science, vol. 49, pp. 297-307, 1989.

[9] "SME Robotics," 2016. [Online]. Available: http://www.smerobotics.org/. [Accessed 19 March 2016]. 